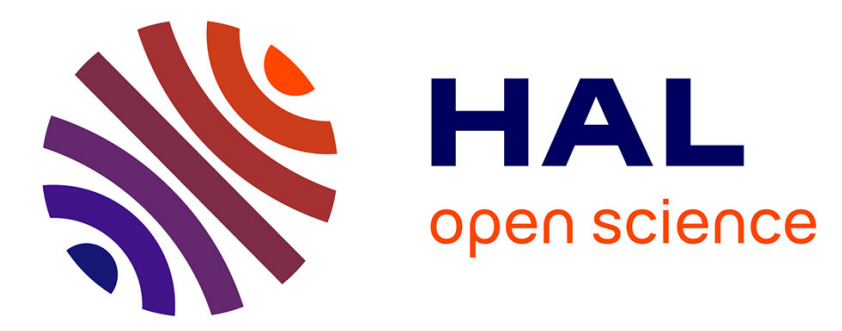

\title{
Towards industrial aero-structural aircraft optimization via coupled-adjoint derivatives
}

\author{
Marco Carini, Christophe Blondeau, Nicolo Fabbiane, Michael Méheut, \\ Mohammad Abu-Zurayk, Johan Feldwisch, Caslav Ilic, Andrei Merle
}

\section{- To cite this version:}

Marco Carini, Christophe Blondeau, Nicolo Fabbiane, Michael Méheut, Mohammad Abu-Zurayk, et al.. Towards industrial aero-structural aircraft optimization via coupled-adjoint derivatives. AIAA AVIATION 2021 FORUM Session: Special Session: MADELEINE Project II, Aug 2021, VIRTUAL EVENT, France. 10.2514/6.2021-3074 . hal-03451068

\section{HAL Id: hal-03451068 \\ https://hal.science/hal-03451068}

Submitted on 26 Nov 2021

HAL is a multi-disciplinary open access archive for the deposit and dissemination of scientific research documents, whether they are published or not. The documents may come from teaching and research institutions in France or abroad, or from public or private research centers.
L'archive ouverte pluridisciplinaire HAL, est destinée au dépôt et à la diffusion de documents scientifiques de niveau recherche, publiés ou non, émanant des établissements d'enseignement et de recherche français ou étrangers, des laboratoires publics ou privés. 


\title{
Towards industrial aero-structural aircraft optimization via coupled-adjoint derivatives
}

\author{
M. Carini ${ }^{1}$, C. Blondeau ${ }^{2}$, N. Fabbiane ${ }^{3}$ and M. Méheut ${ }^{4}$ \\ ONERA The French Aerospace Lab, Meudon, F-92140, France
}

\author{
M. Abu-Zurayk ${ }^{5}$, J. Feldwisch ${ }^{6}$, C. Ilic ${ }^{7}$ and A. Merle ${ }^{8}$ \\ German Aerospace Center (DLR), Germany
}

\begin{abstract}
The paper provides an overview of the maturity achieved on multi-disciplinary aeroelastic and aero-structural adjoint solvers developed during the European project MADELEINE. The main focus is on relevant industrial test cases specified by Airbus based on the XRF-1 research model, representative of a large passenger aircraft. First a crossvalidation of the computed sensitivities among project partners is presented for both rigid and flexible shape gradients, based on a shared wing shape parametrization prescribed by the aircraft manufacturer. Then studies are performed by considering an increased number of parameters representative of industrial design applications. The benefit of taking flexibility into account is demonstrated by comparing single and multi-point optimization results. Finally an outlook of ongoing aero-structural optimizations exploiting an integrated multidisciplinary feasible strategy is provided.
\end{abstract}

\section{Nomenclature}

$C D_{p} \quad=$ pressure drag coefficient

$C D_{f} \quad=$ friction drag coefficient

$C D_{f f} \quad=$ far-field drag coefficient

$C D_{w} \quad=$ wave drag coefficient

$C D_{v p} \quad=$ viscous-pressure drag coefficient

$C D_{i} \quad=$ induced drag coefficient

$C L=$ lift coefficient

$C_{p} \quad=$ pressure coefficient

AoA $=$ Angle of attack

RANS = Reynolds Averaged Navier-Stokes

$C F D=$ Computational Fluid Dynamics

$C A D=$ Computer Aided Design

$F E M=$ Finite Element Method

$X R F-1=$ eXternal Research Forum

\footnotetext{
${ }^{1}$ Dr. Research Engineer, Aerodynamics, Aeroelasticity and Acoustics Dept. marco.carini@onera.fr.

${ }^{2}$ Senior Research Scientist, Aerodynamics, Aeroelasticity and Acoustics Dept. christophe.blondeau@onera.fr.

${ }^{3}$ Dr. Research Scientist, Aerodynamics, Aeroelasticity and Acoustics Dept. nicolo.fabbiane@onera.fr.

${ }^{4}$ Dr. Research Scientist, Aerodynamics, Aeroelasticity and Acoustics Dept. michael.meheut@onera.fr.

${ }^{5}$ Research Engineer, Institute of Aerodynamics and Flow Technologies, Mohammad.Abu-zurayk@dlr.de.

${ }^{6}$ Research Engineer, Institute of aeroelasticity, Johan.Feldwisch@dlr.de.

${ }^{7}$ Research Engineer, Institute of Aerodynamics and Flow Technologies, Caslav.Ilic@dlr.de.

${ }^{8}$ Research Engineer, Institute of Aerodynamics and Flow Technologies, Andrei.Merle@dlr.de.
} 


\section{Introduction}

The industrial practice of High-Fidelity $(\mathrm{HiFi})$ aircraft optimization studies still relies on separated disciplines which also results as a legacy of the internal organization of most companies. However the challenges introduced by new aircraft configurations able to exploit the synergies among aerodynamic, structure and propulsion, critically question this design paradigm, with the need for coupled analysis approaches in order to properly identify the associated multi-disciplinary trade-offs. A typical example is represented by the aerodynamic shape optimization taking into account aero-structural deformation, which becomes crucial to effectively tackle the design of highly flexible wing.

In the companion paper [10], 'frozen-flexibility' approaches are presented to circumvent the computational cost and complexity of a fully-differentiated aero-elastic process while taking into account structural deformation within the aerodynamic shape optimization loop. More precisely, the jig to flight shape deformations are first precomputed for each considered flight condition by performing fully-coupled aeroelastic computations on the baseline geometry. Then a classical multipoint "rigid" adjoint-based aerodynamic optimization is performed on the jig shape, augmented by the baseline structural displacements.

These weakly-coupled approaches although computationally attractive, especially for industrial configurations, neglect the dependency of the structural deformations on aerodynamic shape changes during the optimization process. Indeed the strongly coupled approach requires a fully-differentiated aeroelastic formulation leading to the so-called coupled-adjoint capability [1,7]. Such approaches have been successfully demonstrated in several studies to conduct aeroelastic and aerostructural [2-7] optimizations but often restricted to academic configurations.

Starting from this state-of-the-art, the present paper aims at leveraging current industrial practices by investigating the benefit of a strongly coupled adjoint approach for aero-structural optimization while addressing the full complexity of industry-driven test cases.

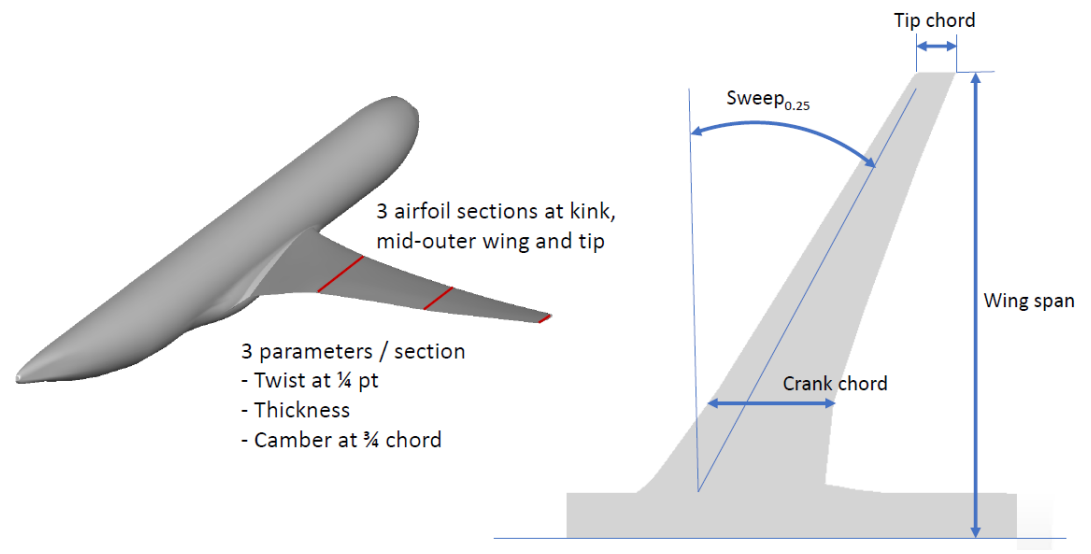

Fig. 1 XRF1 wing-body parametrization for aerodynamic and aeroelastic shape sensitivity validation.

\section{Methodology and Validation}

Before performing complex optimizations, all partners agreed first to validate the implementation of multidisciplinary adjoint formulations on the considered XRF-1 wing-body configuration. In order to ease the validation of sensitivities among partners, some data and disciplinary bricks have been shared whenever possible. For validation purpose all partners adopted a common simplified wing shape parametrization consisting of 13 parameters representative of a typical wing optimization study. Global planform variables like span and sweep as well as local aerofoil parameters like chord, camber, thickness and twist are considered altogether (see Fig. 1). The number of parameters is for sure too low for wing optimization, but is used here for demonstration and validation purpose only. 
Multi-disciplinary optimizations are then performed on a larger set of parameters to demonstrate to benefit of the adjoint approach.

\section{A. Analysis Models}

The steady aeroelastic analysis and sensitivity computation processes require of course a structural model to take flexibility effects into account. As already mention, partners agreed to share the structural model provided by DLR for the gradient validation task. The strategy for generating the structural and the associated aerodynamic grids relies on a common parametric CAD model. This CAD model is also differentiated with respect to the shape design variables, thus providing analytical derivatives leading to a fully consistent adjoint workflow. From this reference outer-mold shape, the finite element model is automatically generated by the CPACS-MoNa ${ }^{9,10}$ process. A series of sections is extracted from the common wing geometry and provided to MoNa following DLR's requirements. These profile slices are merged with a CPACS file for the DLR-XRF1 configuration. From there, the CPACS-MoNa process:

- reads the outer shape;

- creates parametric models for each component;

- discretizes the load carrying structure

- distributes masses;

- creates different mass configurations;

- generates an aerodynamic model;

- analyzes a large set of load cases and selects the critical ones for the structural optimization of each component.

A general overview of the MoNa process is shown in Fig. 2. The finite element model typically output by MONA is presented in Fig. 3. It is worthwhile to mention that the MoNa process not only generates an updated structural model topology but also performs a full sizing considering all relevant critical load cases.

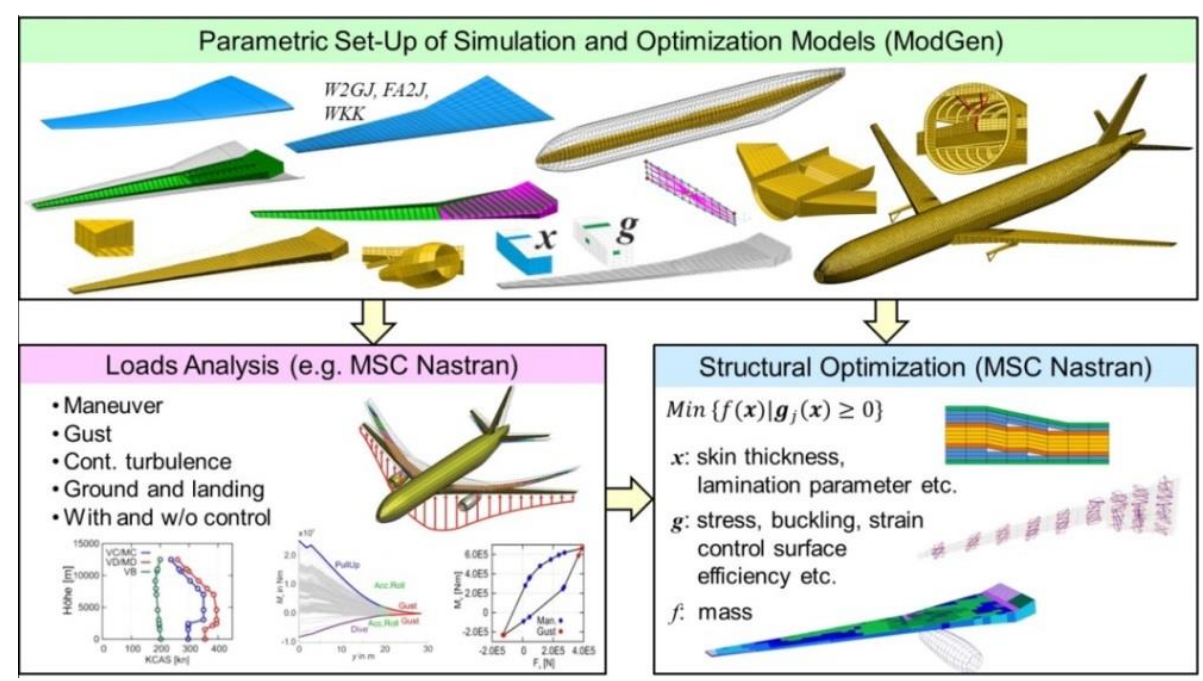

Fig. 2: Core MoNa process at DLR which combines the model generator ModGen and MSC.Nastran.

\footnotetext{
${ }^{9} \mathrm{https}: / / \mathrm{www} . c p a c s . d e /$

${ }^{10}$ https://elib.dlr.de/128099/
} 


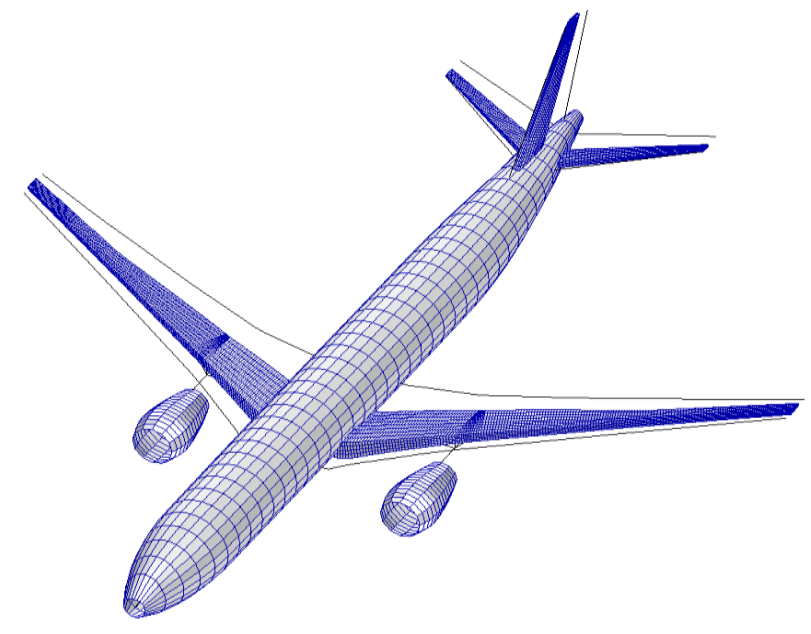

Fig. 3 Aircraft finite element model of the XRF1 test case.
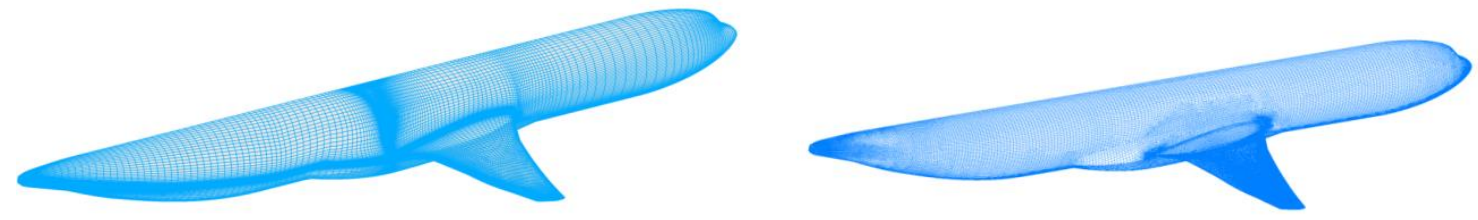

Fig. 4: Surface mesh of the structured (left) and unstructured (right) grids used for gradient validation.

\section{B. Sensitivity Validation}

At first, partners compared rigid and flexible gradients computed with their own in-house tools. More precisely all aerodynamic RANS computations have been performed by Airbus and DLR with the CFD solver TAU using an unstructured grid of 5.7M points. At ONERA the elsA solver (ONERA-Airbus-Safran property) [11] has been used with a comparable grid of 5.3M points. The corresponding surface meshes are illustrated in Fig. 4. The one-equation Spalart-Allmaras model has been adopted by all partners for turbulence modelling. It is worth mentioning that all adjoint sensitivities were computed by considering a fully linearized turbulence model. To assess the relevance of this numerical modelling aspect, gradient computations relying on the frozen turbulent viscosity assumption have also been carried out by ONERA. In addition, in order to make this comparison relevant, all sensitivities were first validated internally by central finite differences by all partners.

Representative results of rigid and aeroelastic computations are reported in Fig 5 and Fig. 6, while corresponding sensitivities for pressure drag coefficient $\left(C D_{p}\right)$ are illustrated in Fig. 7 and Fig. 8, respectively. More details on results computed by Airbus are found in the companion paper [10]. All these results are computed at a representative flight condition within the cruise range. Fig 5 compares the Airbus datum flight shape with the flight shape computed by the aeroelastic analysis using the DLR-XRF-1 structural model. It is seen that the wing deflection is lower indicating a stiffer wingbox. However, the pressure coefficient distributions are very similar showing slightly lower pressure levels on the upper side of the recomputed flight shape. 


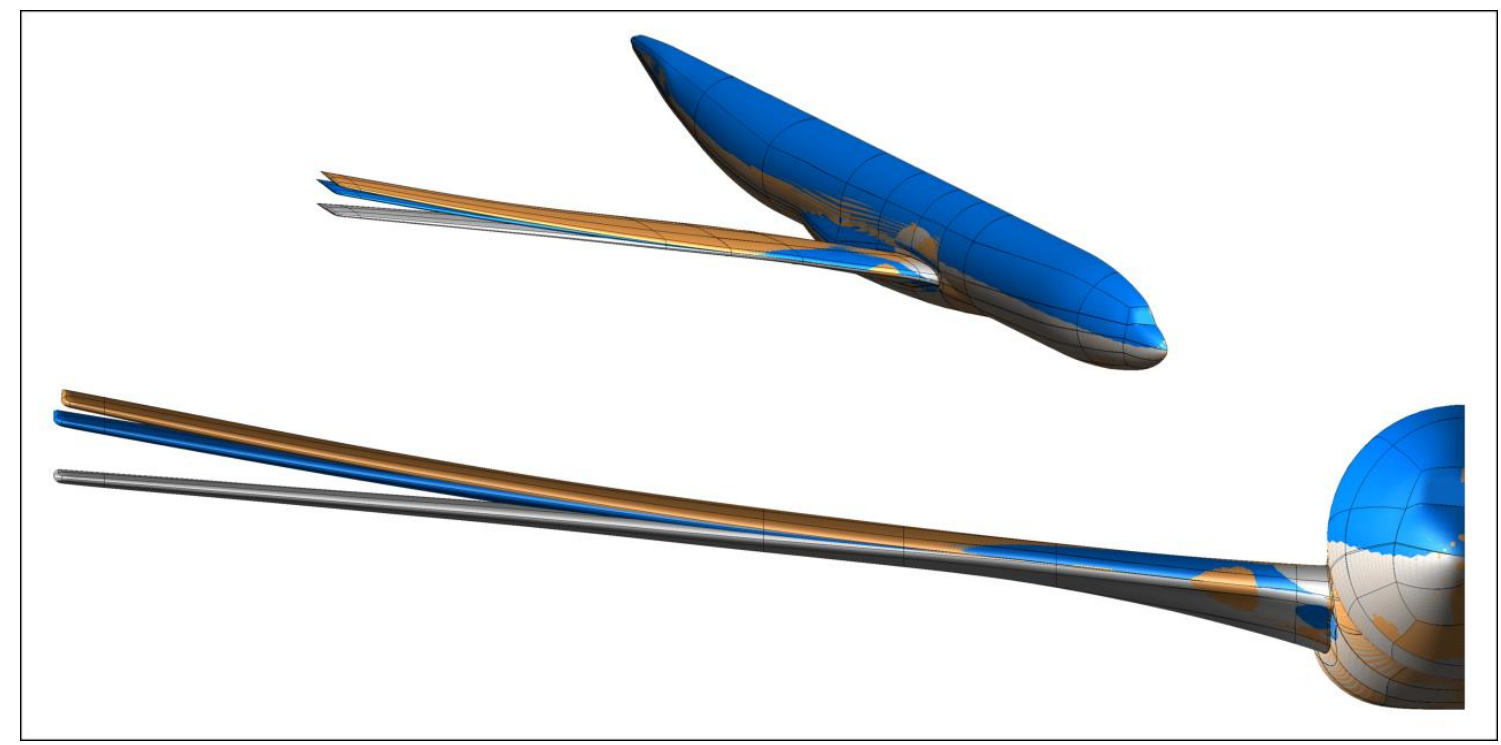

Fig. 5 Flight shape computed by the aeroelastic analysis (blue) versus datum flight shape (Airbus). The datum jig shape is in grey.
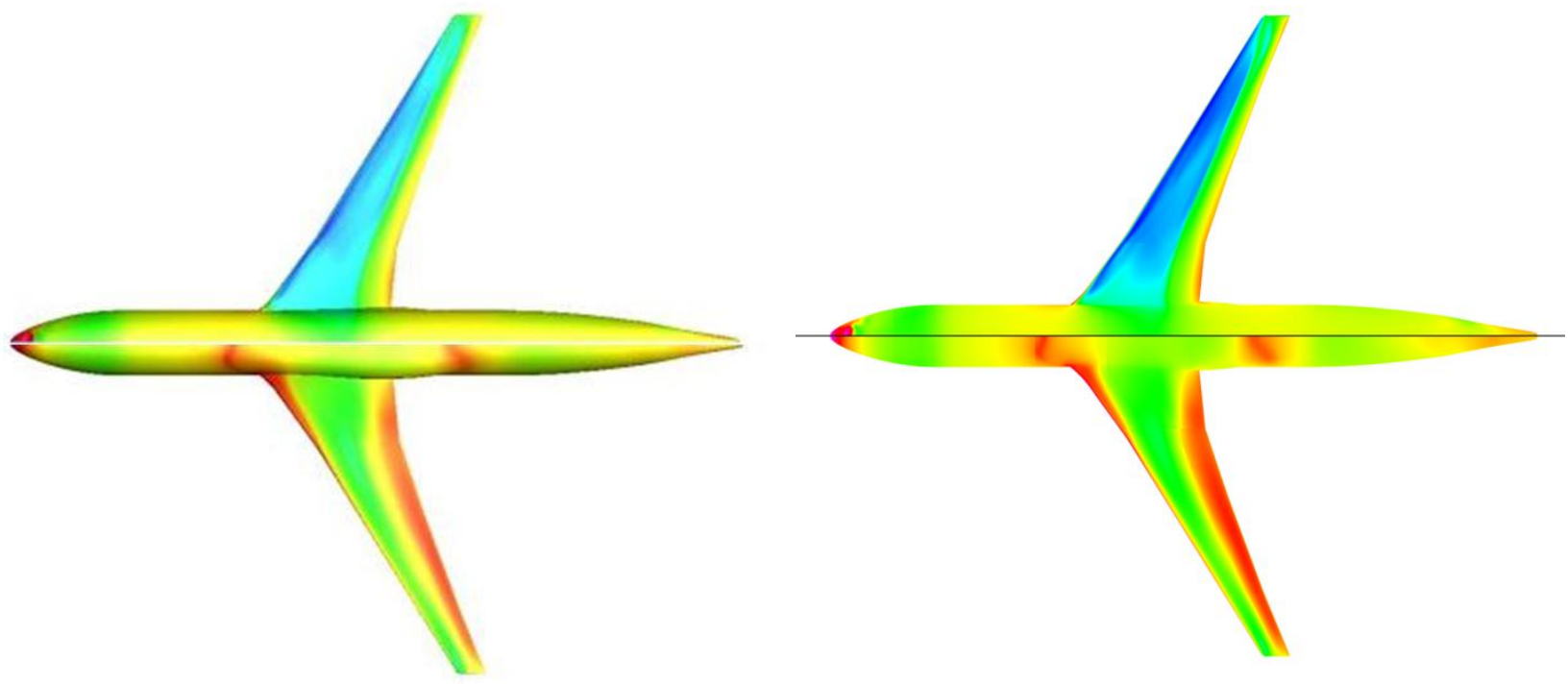

Fig. 6: Pressure coefficient distribution (top = upper surface, bottom = lower surface): comparison between results obtained on the datum flight shape (left) and by the aeroelastic analysis (right).

Note that the rigid sensitivities are computed around the Airbus datum flight shape. The agreement is particularly good, considering variability in meshing strategies, mesh deformation, CFD settings, etc. First it has been observed that the turbulence model linearization can significantly affect the rigid gradient values. However looking at flexible counterparts, this seems to be mitigated by the impact of structural flexibility, which is particularly important for planform design variables.

From this cross-validation exercise partners were sufficiently confident in the maturity and accuracy of their coupled adjoint solvers which was an important milestone before addressing realistic optimizations. 


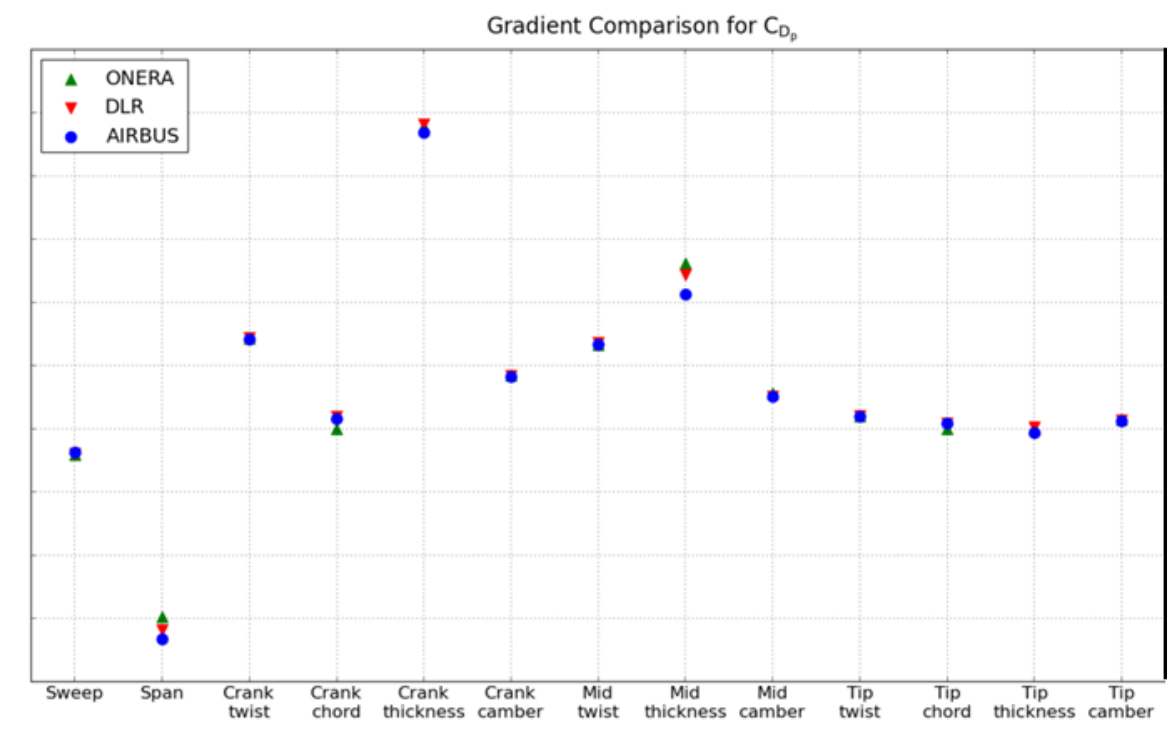

Fig. 7: Comparison of $\mathrm{CD}_{\mathrm{p}}$ sensitivities among ONERA, DLR and AIRBUS on XRF-1 rigid flight shape.

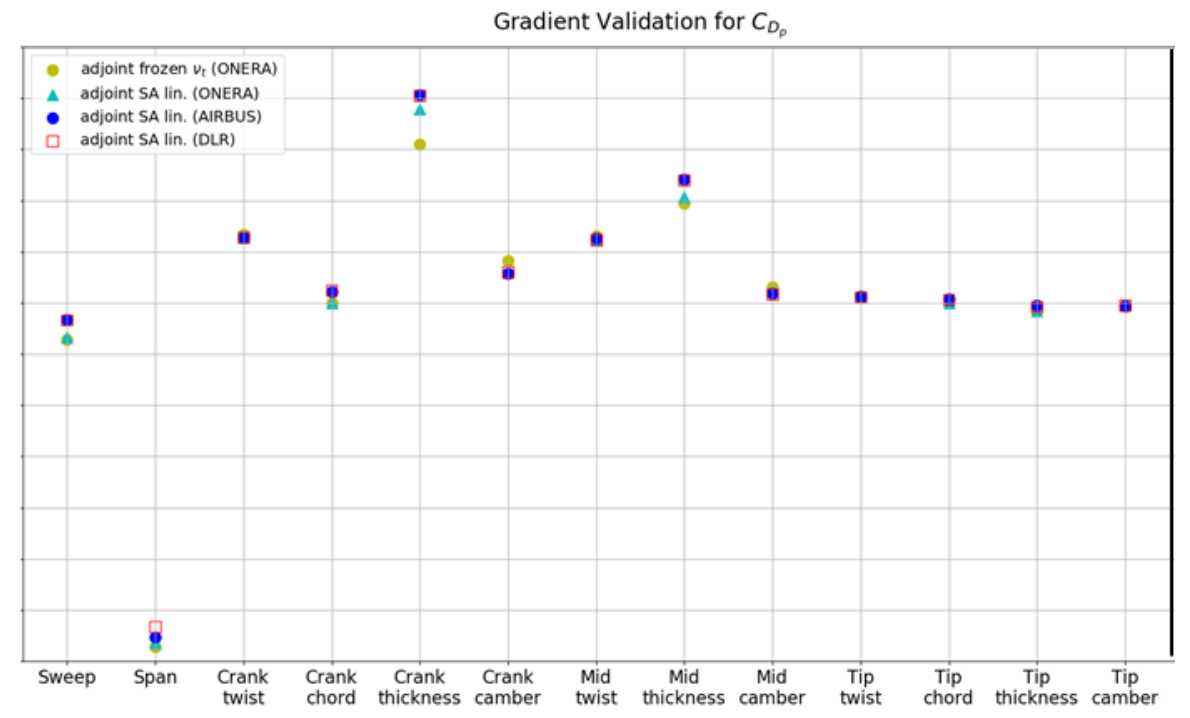

Fig. 8: Comparison of $\mathrm{CD}_{\mathrm{p}}$ sensitivities among ONERA, DLR and AIRBUS on XRF-1 flexible shape.

\section{Optimization results}

\section{A. Enriched shape parametrization}

A refined wing shape parametrization is here introduced to carry out realistic industrial demonstrations of rigid and flexible optimization with frozen wing planform using the elsA solver and two different DLR XRF-1 structural models. In order to enable a finer control of the local airfoil camber and of the twist distribution, the considered parametrization consists of 12 wing sections, as highlighted in Fig. 9. For each section, 6 camber control points are employed which are located at the $15 \%, 30 \%, 45 \%, 60 \%, 80 \%$ and $90 \%$ of the chord. An example of the local 
camber variation that can be prescribed is illustrated in the right upper panel of Fig. 9. The twist distribution is linearly interpolated between the crank and the tip sections (as illustrated in the right middle panel of Fig. 9) with an additive smooth correction by means of a third-order spline for the inner and outer wing, separately (as illustrated in the right lower panel of Fig. 9). The corresponding total number of shape design variables is of 73.

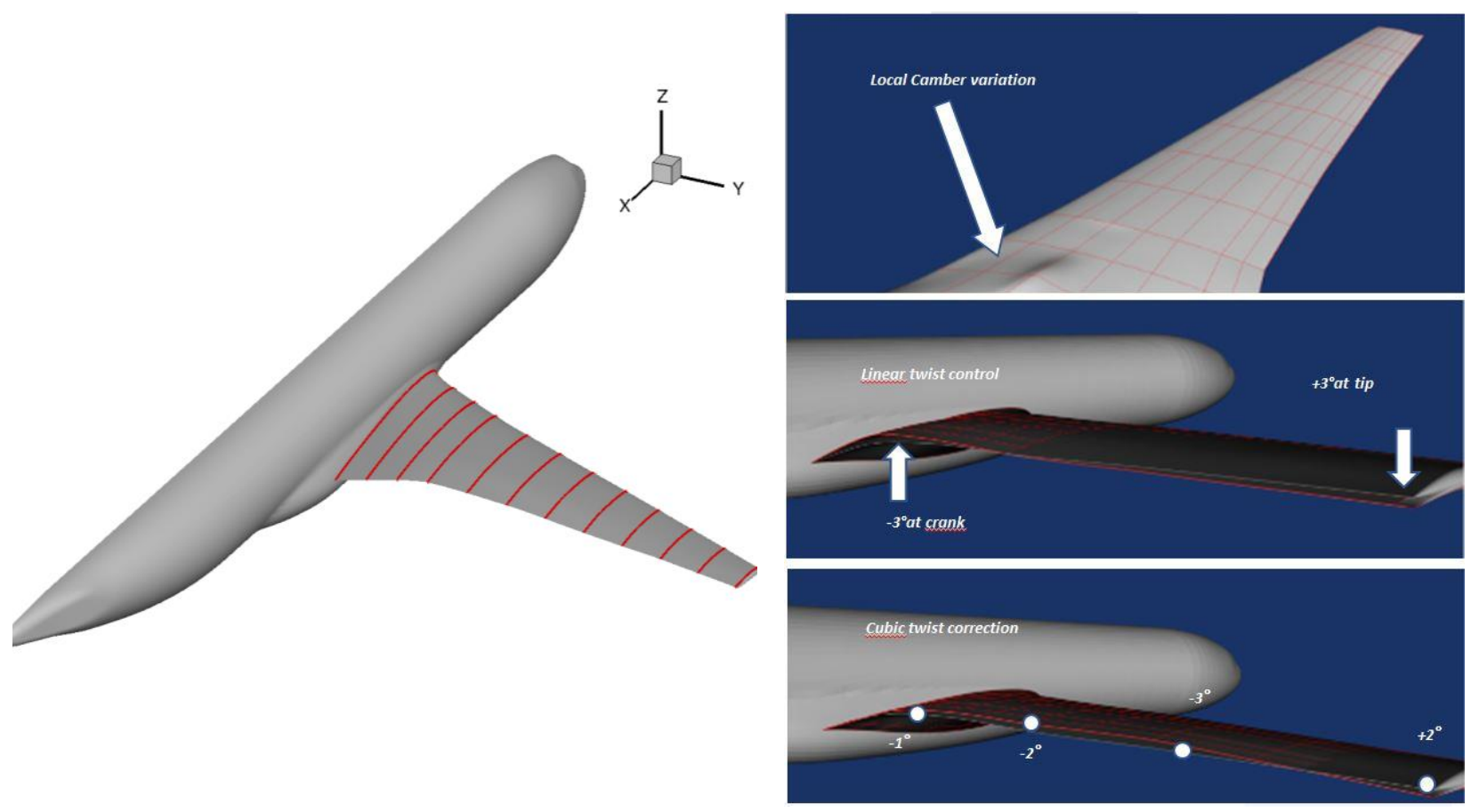

Fig. 9: Enriched XRF-1 wing-shape parametrization: control section locations (left) and examples of camber and twist modifications.

\section{B. Structural models}

Two different structural models FS1 and FS2 have been generated by using CPACS-MoNa. One major difference to the XRF-1 reference structure is a switch to aluminium for the load carrying structure instead of composite materials. Furthermore, titanium is used where the landing gear and the engine are integrated. While for the upper skin, a Young's modulus of 71.0 GPa is used, the lower wing, the ribs and spars are made of a stiffer metal with a Young's modulus of $73.8 \mathrm{GPa}$. The elements of the structural models, like the beams and the shell elements are defined in a preliminary sizing step, where an equivalent wing model is analytically sized using cut-loads. Thus, the preliminary sizing step provides an initial design. The shell-element thicknesses are optimized by minimizing the structural mass using MSC.Nastran. The wing's sub-components are partitioned into 392 design fields, split between ribs, spars, and skins, where each design value controls within its field the wall-thickness property for all shell elements (see Fig. 20). Each design value is constrained by lower and upper bound, which are determined by the preliminary sizing step, considering a minimum wall thickness of at least $2 \mathrm{~mm}$. Maximum von Mises stress, and maximum shear stress of the elements in each design field are constrained by upper and lower bounds. The minor principle stress of each design field is used in an analytical equation to account for buckling constraints.

The load cases used for the structural optimization are different between the two models (FS1 and FS2). In both cases, the loads are computed by the aeroelastic analysis SOL144 and SOL146 of MSC.Nastran. For the FS1 model, pull-up, push-down and roll maneuvres are computed for all mass configurations, which have been suggested by Airbus. For the FS2 model different load cases consisting of six pull-up-, four push-down-, 18 roll-maneuvers and five gusts encounters are investigated, from which only three pull-up, four push-down and two roll-maneuvers appear to be critical and are then used in the structural optimization. The optimum wall thicknesses for the different subcomponents of the FS2 model are shown in Fig. 10. As the FS1 model investigates a larger set of load cases, 
higher critical loads were found for the structural design, which yielded a stiffer design compared to the FS2 model as highlighted by the comparison of the corresponding flight shapes in Fig. 11.

The final structural mass of the wingbox for the model FS1 is $18,947 \mathrm{~kg}$ and it is of $18,724 \mathrm{~kg}$ for the more flexible model FS2.

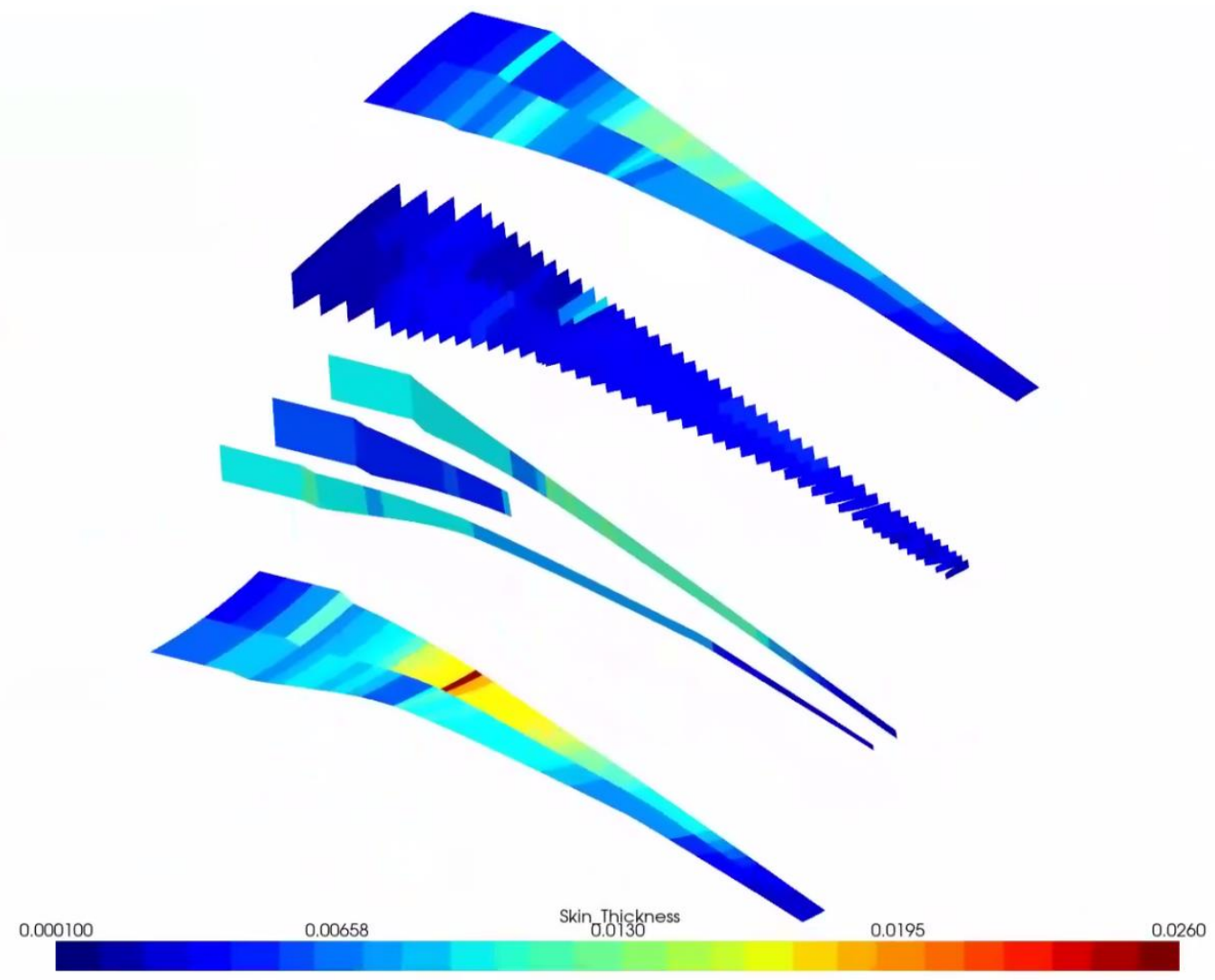

Fig. 10. Wall thickness distribution of the final FS2 model, which is based on a smaller set of load cases. 


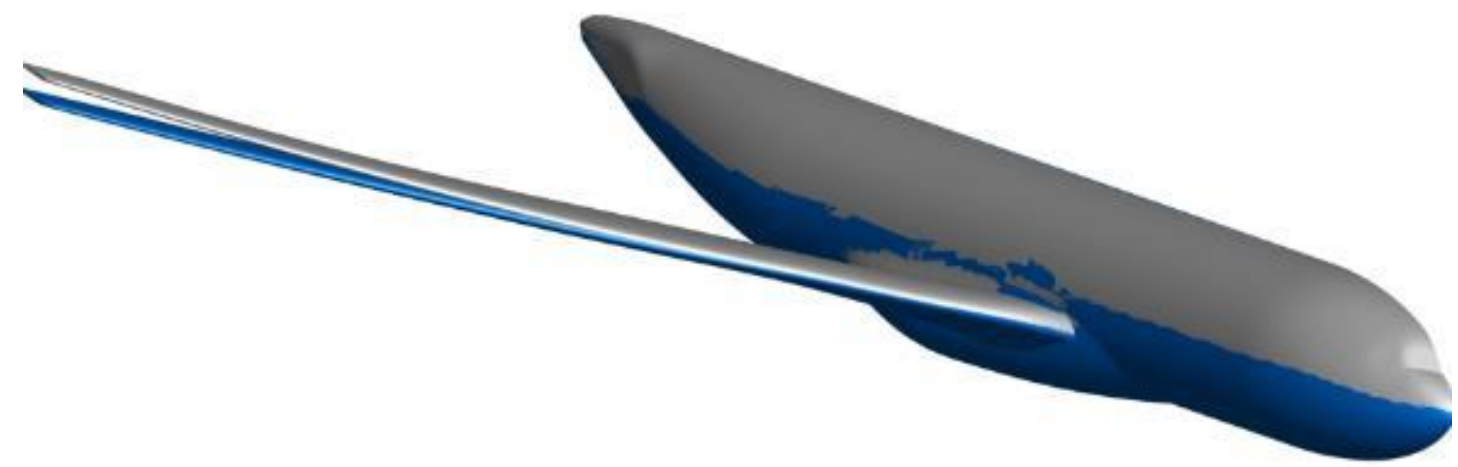

Fig. 11 Baseline flight shapes resulting from aeroelastic computation around the XRF-1 baseline jig-shape: the flight shape (blue shaded surface) corresponds to the stiffer FS1 structural model and the flight shape (gray shaded surface) corresponds to the more flexible FS2 structural model.

\section{Comparison of rigid and flexible optimization}

A first optimization demo is presented here by considering the minimisation of the far-field drag coefficient of the aircraft [8] at the same flight conditions considered for the validation task with $C L=0.5$. The angle of attack (AoA) is introduced as an additional variable in the optimization process, for a total of 74 variables. Both rigid and flexible approaches are considered for such purpose. More precisely for the rigid case the parametrization is defined directly on the baseline flight shape while for the flexible case, the parametrization is associated with the baseline jig shape. However it is worth to note that the flight and the jig baseline shapes are fully consistent, since the first one has been obtained as part of the solution of the aeroelastic computation on the second one at the considered target lift. For both the two considered structural models, i.e. FS1 and FS2, rigid and flexible optimization convergence histories are summarised in Fig. 12 by showing the percentage gain on the cost function and the prescribed lift constraint. The resulting gain on the drag coefficient increases from $\sim 3.5-3.7 \%$ for the FS1 test case to $\sim 4.7-5.0 \%$ for the FS2 test case, which features a more flexible wing structure. A more detailed analysis of the optimum gains is presented in Table 1 by inspecting the related far-field drag breakdown. For the pure aerodynamic optimized shapes, corrected performance obtained by re-computing a posteriori the structural deflection, have also been reported. For both test cases the gain associated with the pure aerodynamic optimization is slightly higher compared to the value achieved by aeroelastic optimization. However when the aeroelastic correction is introduced on the rigid results, the two performances get very close, which is furthermore confirmed by inspecting the different drag components, except for the wave drag of the FS1 case. As expected, the wave-drag component is the most affected one by the optimization process. Pressure coefficient distributions are compared in 

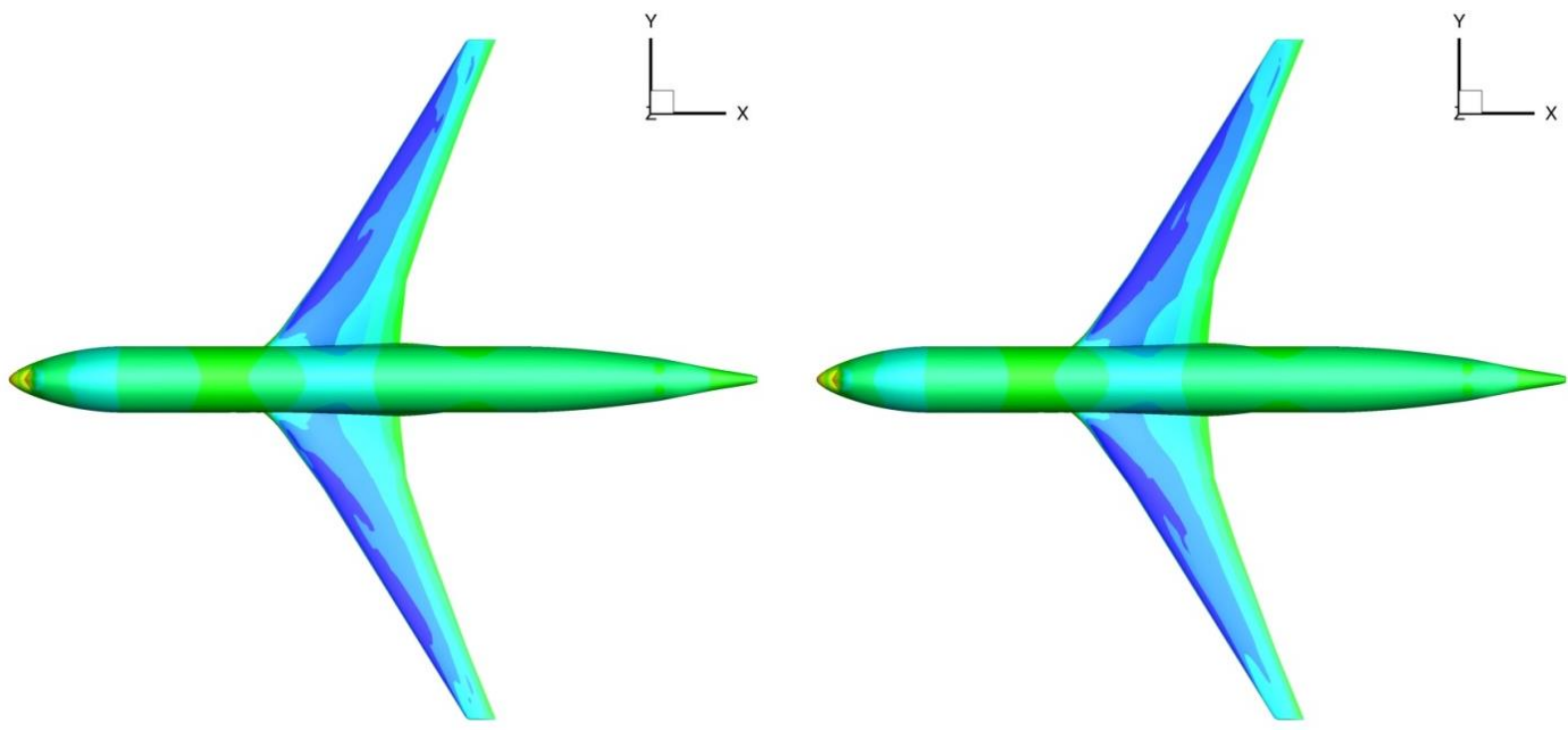

Fig. 13 for the suction side only: remarkable differences among rigid and flexible results are observed for the FS2 test case on the outboard wing. Corresponding spanwise load and drag distributions are reported and compared in
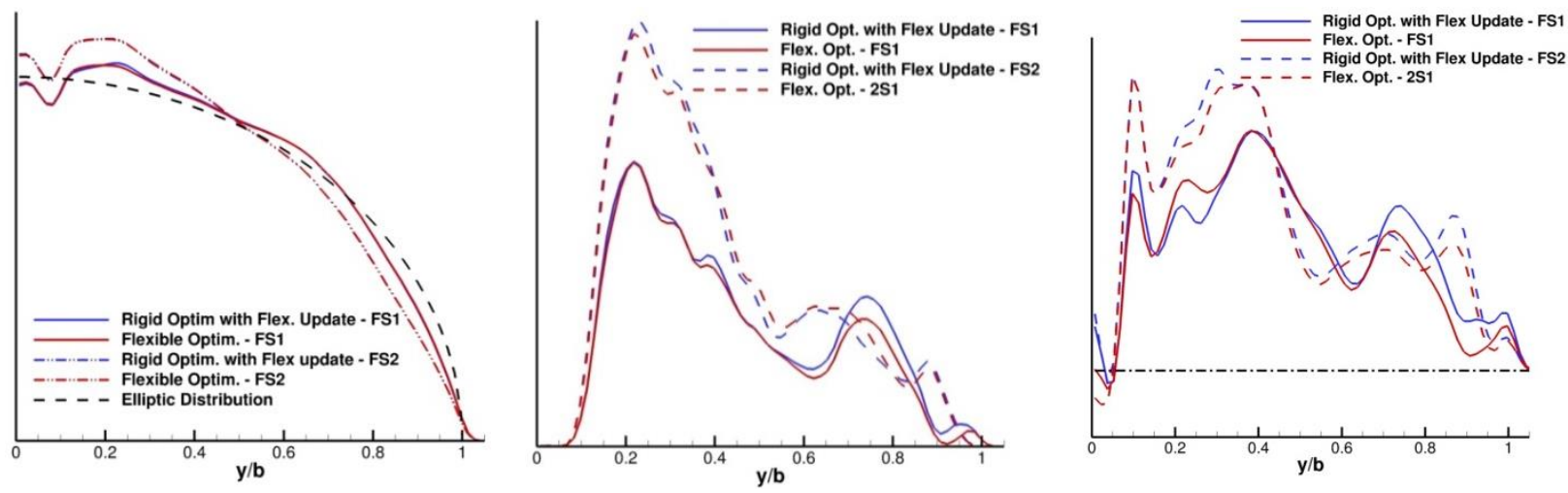

Fig. 14. Deviation from the elliptic distribution results higher in the FS2 case with an increase of the aerodynamic load in the inboard region and a decrease in the outboard one. At the same time only negligible differences are observed among rigid and flexible results for both test cases. Similar considerations hold for the viscous-pressure drag distribution, while differences are more pronounced for wave drag, more precisely on the outboard region for the FS1 case and on the inboard region for the FS2 case. For both case, the variations with respect to the baseline distribution clearly shows that drag reduction is not limited to a particular span interval but occurs along the whole wing span. In particular, the achieved wave-drag reduction is larger for the FS2 compared to the FS1 one. 

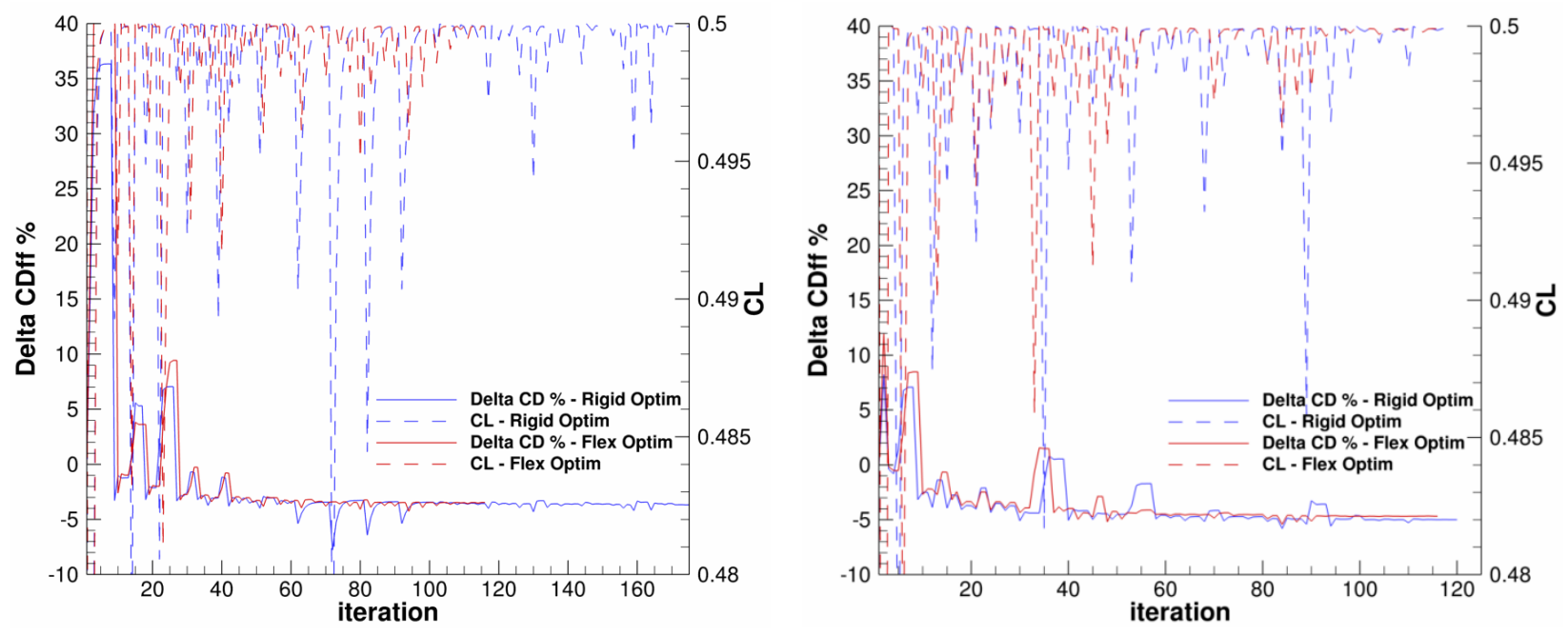

Fig. 12 Rigid and flexible wing shape optimization for FS1 (left) and FS2 (right) test cases: evolution of the far-field drag cost-function and of the lift constraint.

\begin{tabular}{|c|c|c|c|c|c|c|}
\hline Test Case & Optim & $\Delta \mathbf{C D}_{\mathrm{i}}$ & $\Delta \mathbf{C D}_{\mathrm{w}}$ & $\Delta \mathbf{C D}_{\mathrm{vp}}$ & $\Delta \mathbf{C D}_{\mathrm{f}}$ & $\Delta \mathbf{C D}_{\mathrm{ff}}$ \\
\hline \multirow{3}{*}{ FS1 } & Rigid & $+1.00 \%$ & $-90.44 \%$ & $-6.66 \%$ & $+0.38 \%$ & $-3.68 \%$ \\
\hline & $\begin{array}{c}\text { Rigid with } \\
\text { flexible } \\
\text { update }\end{array}$ & $+0.72 \%$ & $-90.40 \%$ & $-6.02 \%$ & $+0.61 \%$ & $-3.61 \%$ \\
\hline & Flexible & $+0.70 \%$ & $-84.81 \%$ & $-6.08 \%$ & $+0.49 \%$ & $-3.47 \%$ \\
\hline \multirow{3}{*}{ FS2 } & Rigid & $+0.80 \%$ & $-92.45 \%$ & $-7.83 \%$ & $+0.29 \%$ & $-4.95 \%$ \\
\hline & $\begin{array}{c}\text { Rigid with } \\
\text { flexible } \\
\text { update }\end{array}$ & $+1.03 \%$ & $-93.29 \%$ & $-7.77 \%$ & $+0.32 \%$ & $-4.88 \%$ \\
\hline & Flexible & $+1.04 \%$ & $-93.17 \%$ & $-7.15 \%$ & $+0.57 \%$ & $-4.68 \%$ \\
\hline
\end{tabular}

Table 1. Analysis and comparison of rigid and flexible optimization results in terms of far-field drag decomposition for the two considered test cases FS1 and FS2. Results obtained by re-computing the aeroelastic equilibrium around the rigidly optimized flight shapes are denotes as "Rigid with flexible update". 

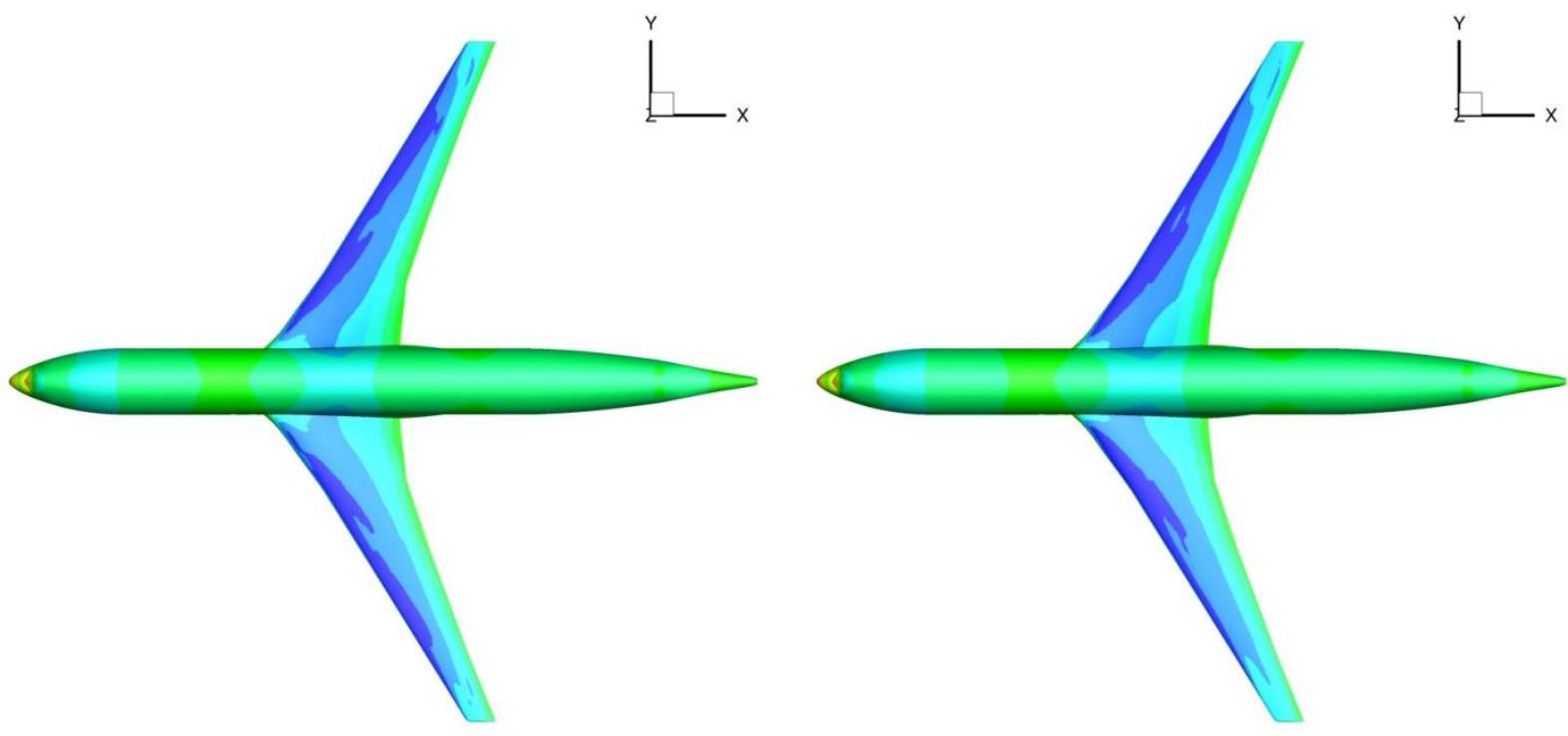

Fig. 13. Comparison of optimized pressure side $C_{p}$ distributions: rigid optimized with flexible update (lower half model) and flexible optimized (upper half model). Results for FS1 (left picture) and FS2 (right picture)

test cases.
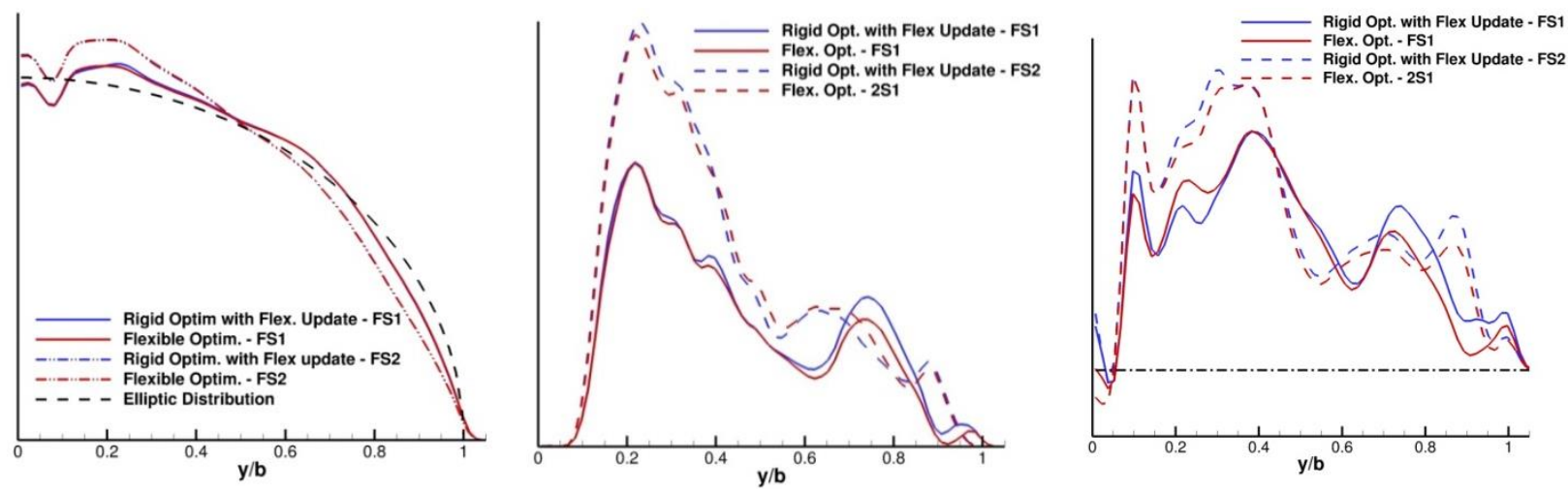

Fig. 14. Comparison of rigid and flexible optimization results: spanload (left panel), wave drag (center panel) and viscous-pressure drag (right panel) for both FS1 and FS2 test cases. Note that drag distribution, correspond to variations with respect to the associated baseline: the vertical axis is reversed so that variation above the zero dashed-dotted line results in a drag reduction with respect the baseline.

\section{Multi-point flexible optimization}

A multi-point (MP) flexible optimization is here presented based on the extension of the FS2 test case to include two additional flight points at $C L=0.45$ and $C L=0.55$. The considered cost function is given by the sum of the far-field drag over the three flight conditions. For each flight condition and consistently with the associated lift constraint, the corresponding value of AoA is introduced as a variable in the optimization process in addition to the 73 shape design variables associated with the wing jig-shape parametrization. The total number of optimization variables is therefore equal to 76. The optimization convergence history is reported in Fig. 15, showing a gain of $\sim 4.1 \%$ on the cost function. A qualitative comparison of the baseline and optimized jig shapes is given in Fig. 16 (left panel) along 
with the evolution of the non-dimensional twist design variables (right panel). In the considered definition of the design space, the twist variables share the same symmetric range of variation around their baseline value, which is made non-dimensional as [0,1]. Therefore, for each twist design variable, the corresponding non-dimensional baseline value is given by 0.5 . As shown in Fig. 16, the optimization leads to increase the twist of all the associated control sections. More precisely, the variation is found more important on the outboard wing compared to the inboard wing. The drag-breakdown analysis of the optimization gain for the three considered flight points is presented in Table 2. In addition, results from the single-point (SP) flexible optimization previously performed at $C L=0.5$ are also provided. These additional results have been obtained by performing aeroelastic computation at $C L=0.45$ and $C L=0.55$ of the optimized FS2 jig shape at $C L=0.5$. As expected, the SP optimized shape is performing slightly better than the MP one at $C L=0.5$, by achieving a higher reduction of the wave-drag component. On the contrary, for the SP case, the performance gain is completely lost when moving to the flight point at lower $C L$ while slightly drops below the MP one at the highest $C L$ value. This confirms the expected benefit from the MP optimization in terms of robustness of the performance gain in the neighborhood of the target cruise condition. The comparison of pressure distributions illustrated in Fig. 17 with the baseline ones (not reported here) shows that the most important differences are located on the suction side of the wing, consistently with leading reduction of the wave drag. Finally, the spanwise distribution of the aerodynamic load as well as of the wave and viscous-pressure drag components are analyzed in Fig. 18 and Fig. 19, respectively. More precisely for the drag components, variations with respect to corresponding baseline distributions are reported. Similarly to what observed for the SP optimizations, for each flight point, positive and negative variation of the aerodynamic load compared to the theoretical elliptic distribution, are found to characterize the inboard and outboard wing regions, respectively. Deviations are also slightly reduced by increasing the lift coefficient from $C L=0.45$ to $C L=0.55$. Consistently with the values reported in Table 2, the inspection of the spanwise drag distributions in Fig. 19 shows that the wave-drag is greatly reduced at $C L=0.5$. At $C L=0.55$ the wave-drag is essentially reduced everywhere along the span but not as much as for $C L=0.5$, while at $C L=0.45$, although globally reduced, a small pocked of increased wave-drag is observed around the mid-span position. The behavior of the viscous-pressure drag contribution (superposed in the same plots) well correlates with the one of the wave-drag, which can be physically interpreted based on the impact of compressible effects on the boundary-layer growth.

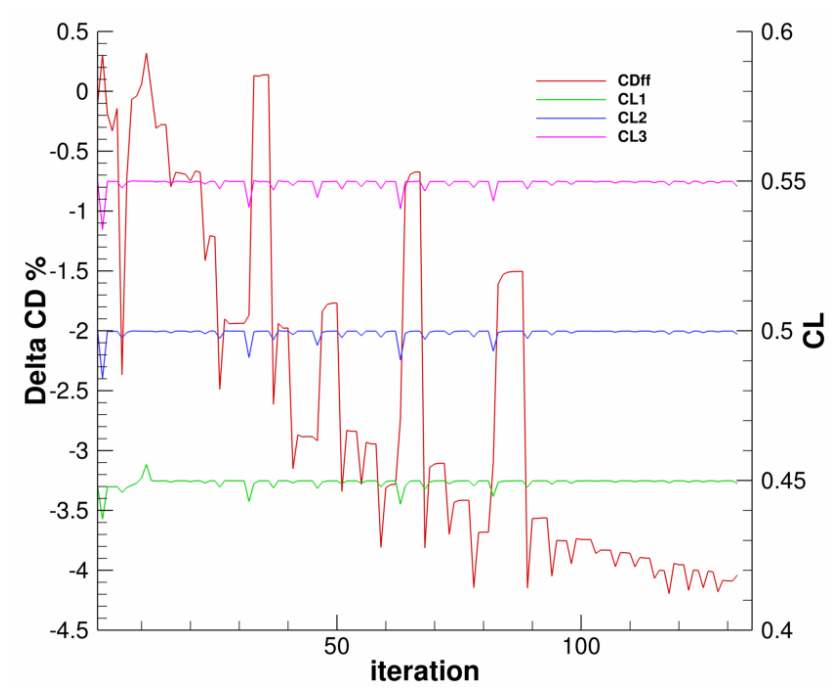

Fig. 15. Multi-point flexible wing shape optimization: evolution of the far-field drag cost-function and of the lift constraints. 

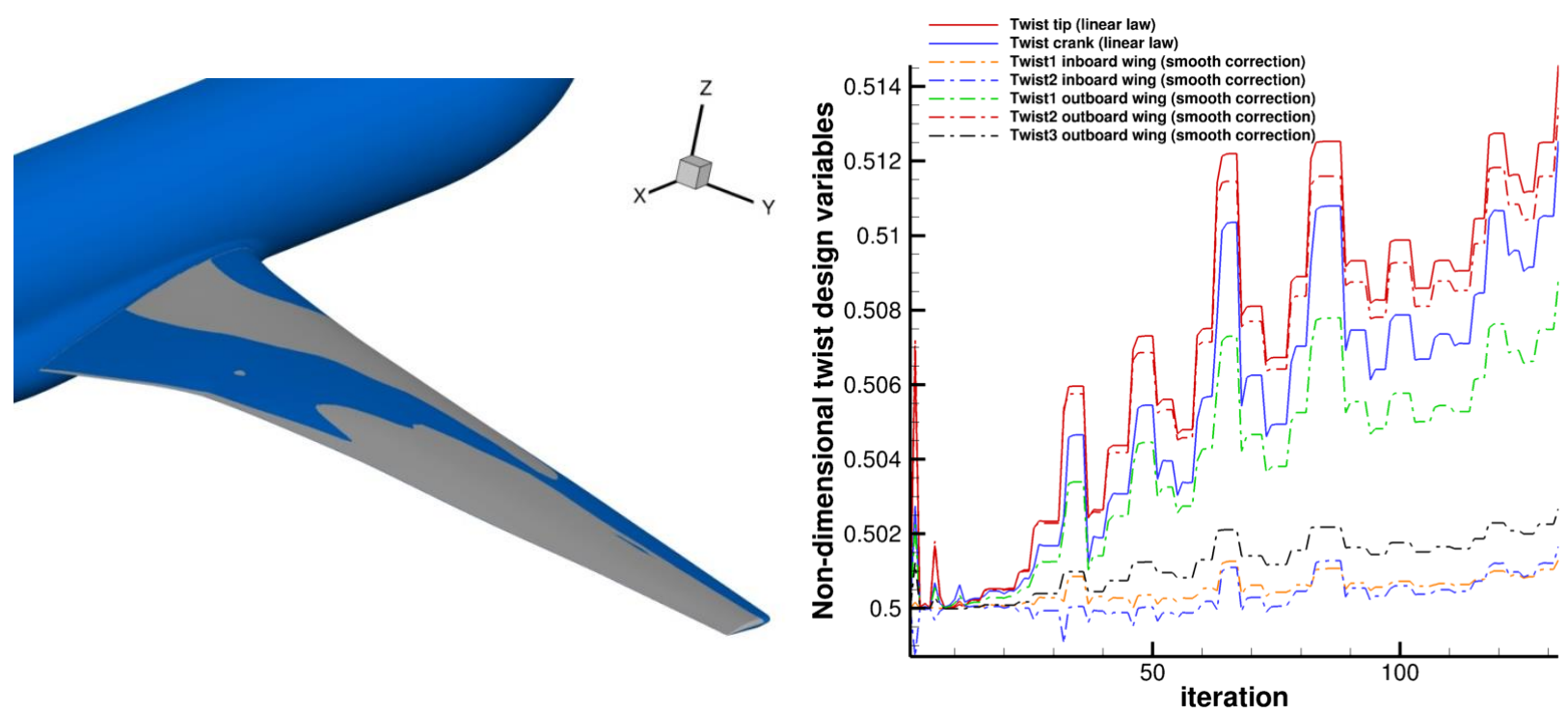

Fig. 16. Qualitative comparison of baseline (gray shaded) and optimized (blue shaded) jig shapes for multipoint flexible optimization (left panel) and optimization history of non-dimensional twist design variables (right panel).

\begin{tabular}{|c|c|c|c|c|c|c|}
\hline Optim & $C L$ & $\triangle C D_{i}$ & $\triangle C D_{w}$ & $\Delta C D_{v p}$ & $\triangle C D_{f}$ & $\Delta C D_{f f}$ \\
\hline \multirow{2}{*}{$\begin{array}{c}\text { MP } \\
\text { SP }\end{array}$} & \multirow{2}{*}{0.45} & $+0.62 \%$ & $-70.91 \%$ & $-4.05 \%$ & $+0.39 \%$ & $-2.61 \%$ \\
\hline & & $+1.36 \%$ & $-7.96 \%$ & $+1.31 \%$ & $+0.23 \%$ & $+0.55 \%$ \\
\hline MP & \multirow{2}{*}{$\mathbf{0 . 5 0}$} & $+0.66 \%$ & $-86.08 \%$ & $-6.58 \%$ & $+0.50 \%$ & $-4.45 \%$ \\
\hline SP & & $+1.04 \%$ & $-93.17 \%$ & $-7.15 \%$ & $+0.57 \%$ & $-4.68 \%$ \\
\hline MP & \multirow{2}{*}{0.55} & $+0.62 \%$ & $-60.35 \%$ & $-5.39 \%$ & $+0.41 \%$ & $-4.93 \%$ \\
\hline SP & & $+1.17 \%$ & $-60.78 \%$ & $-5.02 \%$ & $+0.44 \%$ & $-4.80 \%$ \\
\hline \multicolumn{2}{|c|}{ Obj. function } & \multicolumn{5}{|c|}{$\Delta C D_{f f \mid C L=0.45}+\Delta C D_{f f \mid C L=0.5}+\Delta C D_{f f \mid C L=0.55}$} \\
\hline \multicolumn{2}{|c|}{ MP } & \multicolumn{5}{|c|}{$-4.09 \%$} \\
\hline \multicolumn{2}{|c|}{ SP } & \multicolumn{5}{|c|}{$-3.16 \%$} \\
\hline
\end{tabular}

Table 2. Analysis of multi-point (MP) flexible optimization results in terms of far-field drag decomposition and comparison with the corresponding single-point flexible optimization (SP) results (FS2 test case). 

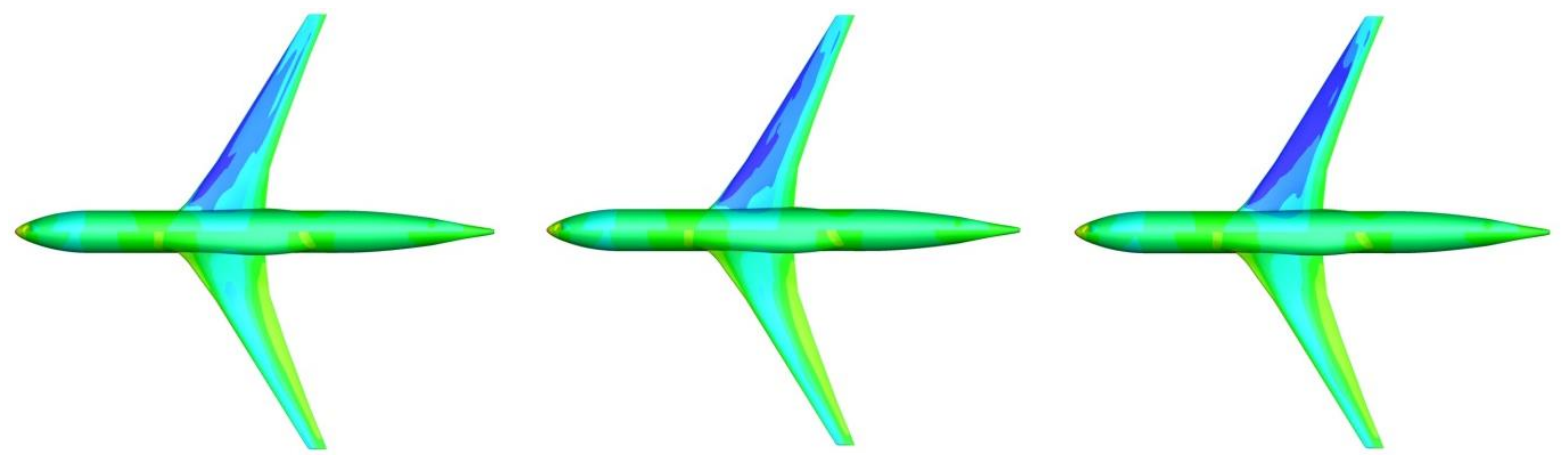

Fig. 17: $C_{p}$ distribution for the multi-point flexible optimized configuration: pressure side (lower half model) and suction side (upper half model). Panel from left to right refer to flight point at $C L=0.45, C L=0.5$ and $C L=0.55$, respectively.
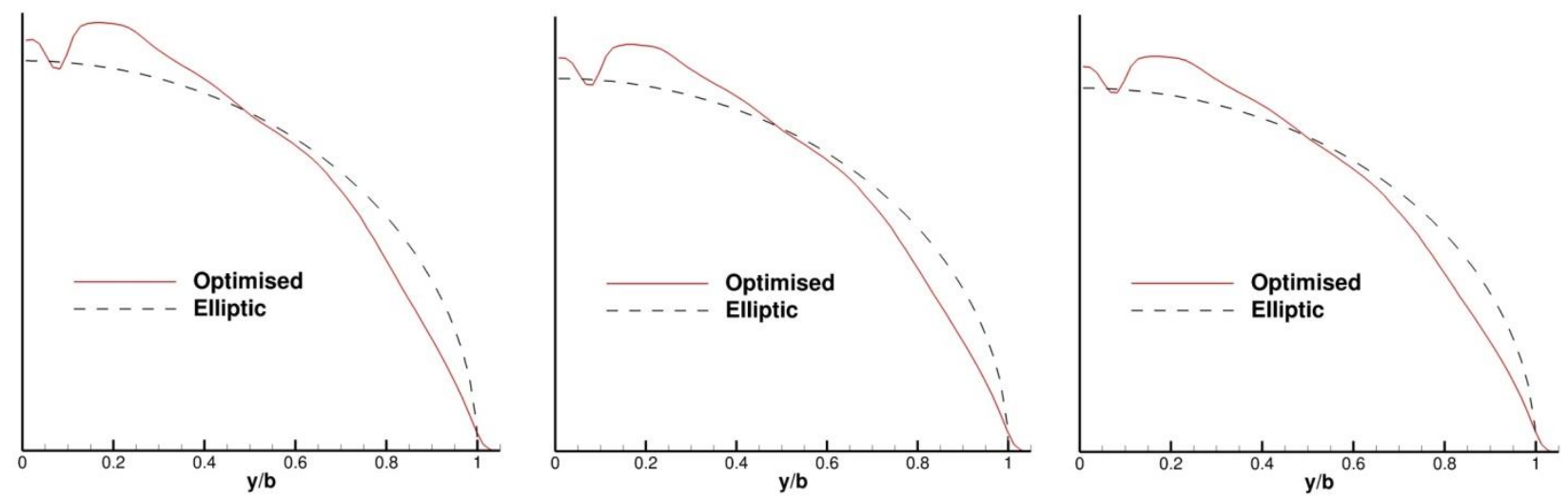

Fig. 18. Comparison of spanload distributions resulting from multi-point flexible optimization. Panel from left to right refer to flight point at $C L=0.45, C L=0.5$ and $C L=0.55$, respectively.
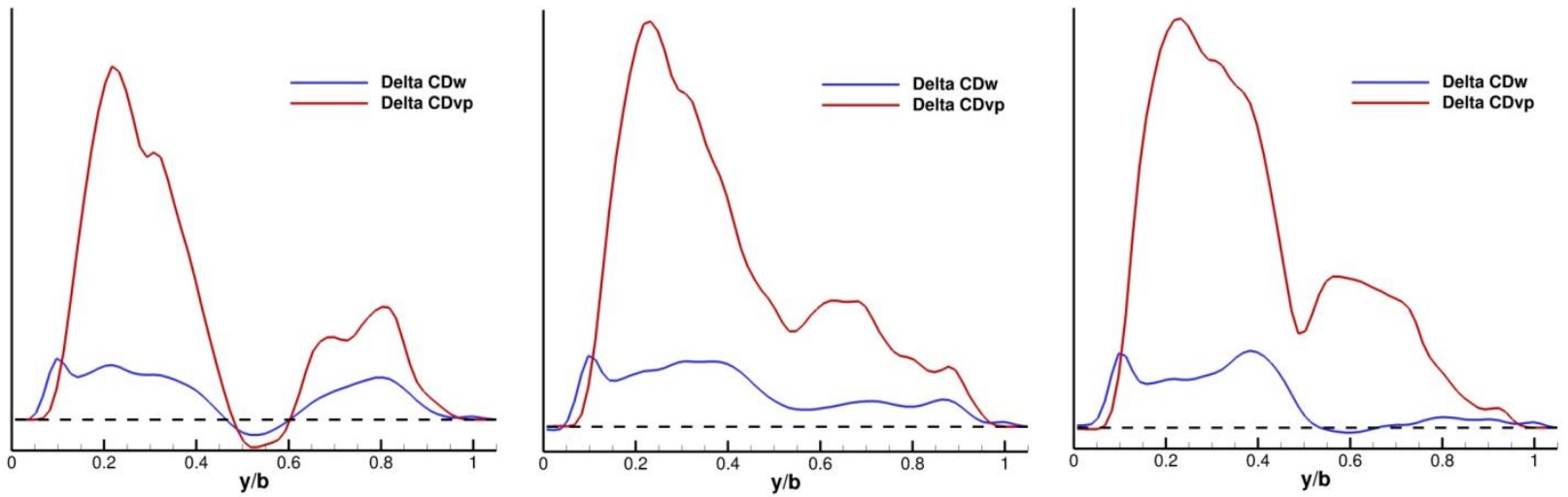

Fig. 19. Variation of wave and viscous-pressure drag distribution with respect to the baseline as obtained from multi-point flexible optimization. Panel from left to right refer to flight point at $C L=0.45, C L=0.5$ and $C L=0.55$, respectively. Note that in these plots the vertical axis is reversed so that variation above the zero dashed line results in a drag reduction with respect to the baseline. 


\section{Ongoing Analyses towards aero-structural optimization}

\section{A. Cross-derivatives of aerodynamic coefficients}

In the previous sections multi-point optimizations have been conducted with the primal objective of assessing the benefit of engaging aeroelastic analyses and associated sensitivity analyses by comparing nominal performances of various optimized configurations. The design variables only affected the airfoil shapes and the twist law while the structural stiffness was considered frozen. This strategy is suitable for an asymmetric aero-structural formulation where for instance the structural design task is converged at the end of each iteration of the outer aerodynamic shape optimization process. The structural model is then updated for the next cycle of aerodynamic shape optimization.

To step further toward a strongly coupled aero-structural optimization process, we now consider the computation of an important cross-derivative term like the sensitivity of any aerodynamic function of interest with respect to structural design variables. In this section we restrict to the specific case where design parameters control the physical properties of the finite element model (skin thicknesses, stringer sections, ...). Therefore, the geometry of the structural grid is not affected by the parametrization. Under these assumptions, the total derivative of an aerodynamic function of interest $J$ with respect to a sizing parameter $p$ is written as [7]:

$$
\frac{d J}{d p}=-\lambda_{s}{ }^{T} \frac{\partial \boldsymbol{K}}{\partial p} \boldsymbol{U},
$$

where $\lambda_{s}$ is the structural adjoint vector, $\boldsymbol{K}$ the stiffness matrix and $\boldsymbol{U}$ the structural displacement vector corresponding to the steady aeroelastic equilibrium under cruise flight conditions. We consider the set of 392 design variables that was previously used for the wingbox sizing process (see section IV.B). The figure below illustrates the distribution of sizing parameters. Each element's physical property in a colored design area is linearly linked to one independent design variable. The structural displacement field for the more flexible FS2 finite element model is plotted in Fig. 21.

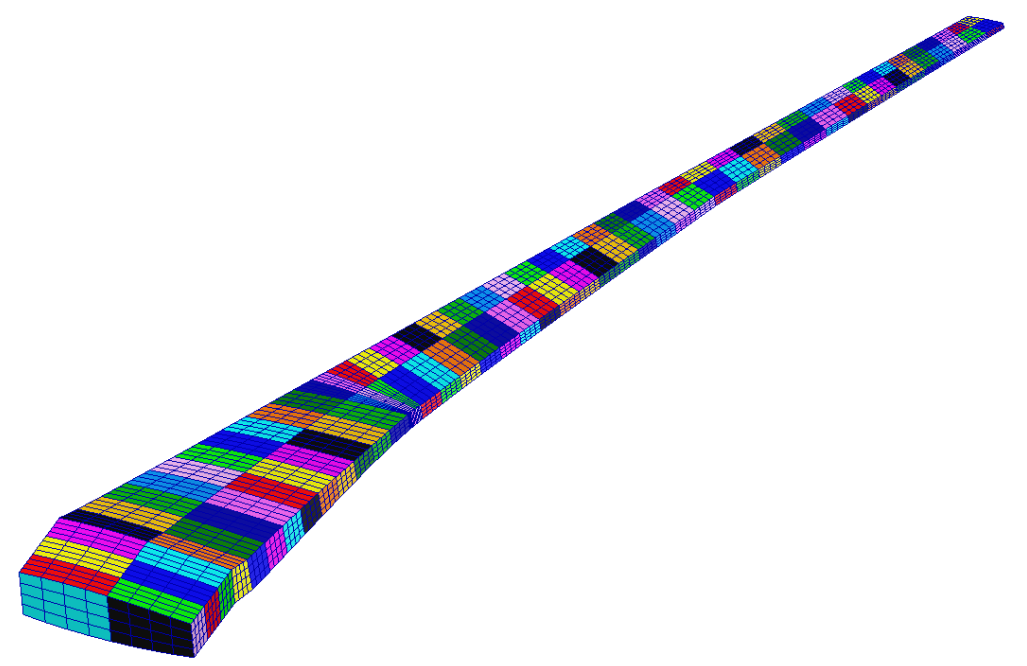

Fig. 20. Definition of the 392 wingbox structural design variables . 


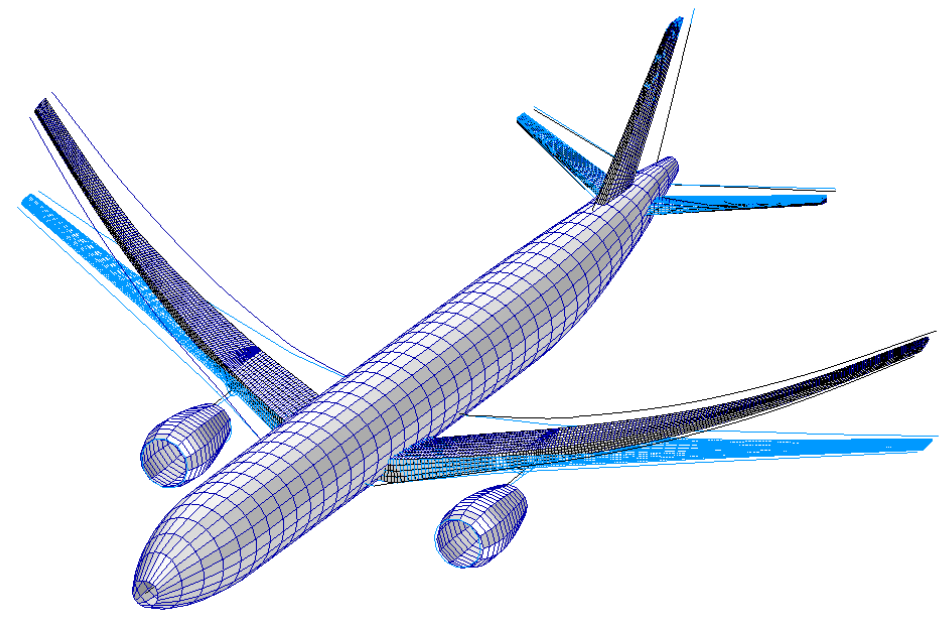

Fig. 21. Nominal cruise flight shape for the more flexible FS2 structural model.

The total derivative $\mathrm{dJ} / \mathrm{dp}$ is then automatically reconstructed from a standard static optimization solution of the commercial MSC.Nastran software using a dedicated DMAP (Direct Matrix Abstraction Programming) alter. The final post-processing step consists in the visualization of the sensitivity map for the aerodynamic coefficient of interest. For illustration, the sensitivity distribution of the pressure drag coefficient is presented in Fig. 22. From this plot a number of observations can be formulated. First the areas associated to negative sensitivity values means that an increase in local stiffness will lead to a decrease of pressure drag coefficient. The larger contribution is located on the rear part of the upper cover. Also a sign change occurs between the light-green and yellow colored areas showing that a stiffness increase of the outer wing box will be detrimental for the pressure drag coefficient. With the addition of this cross-sensitivity contribution the whole sensitivity vector of any aerodynamic function of interest can now be exploited in the high-fidelity optimization process. 


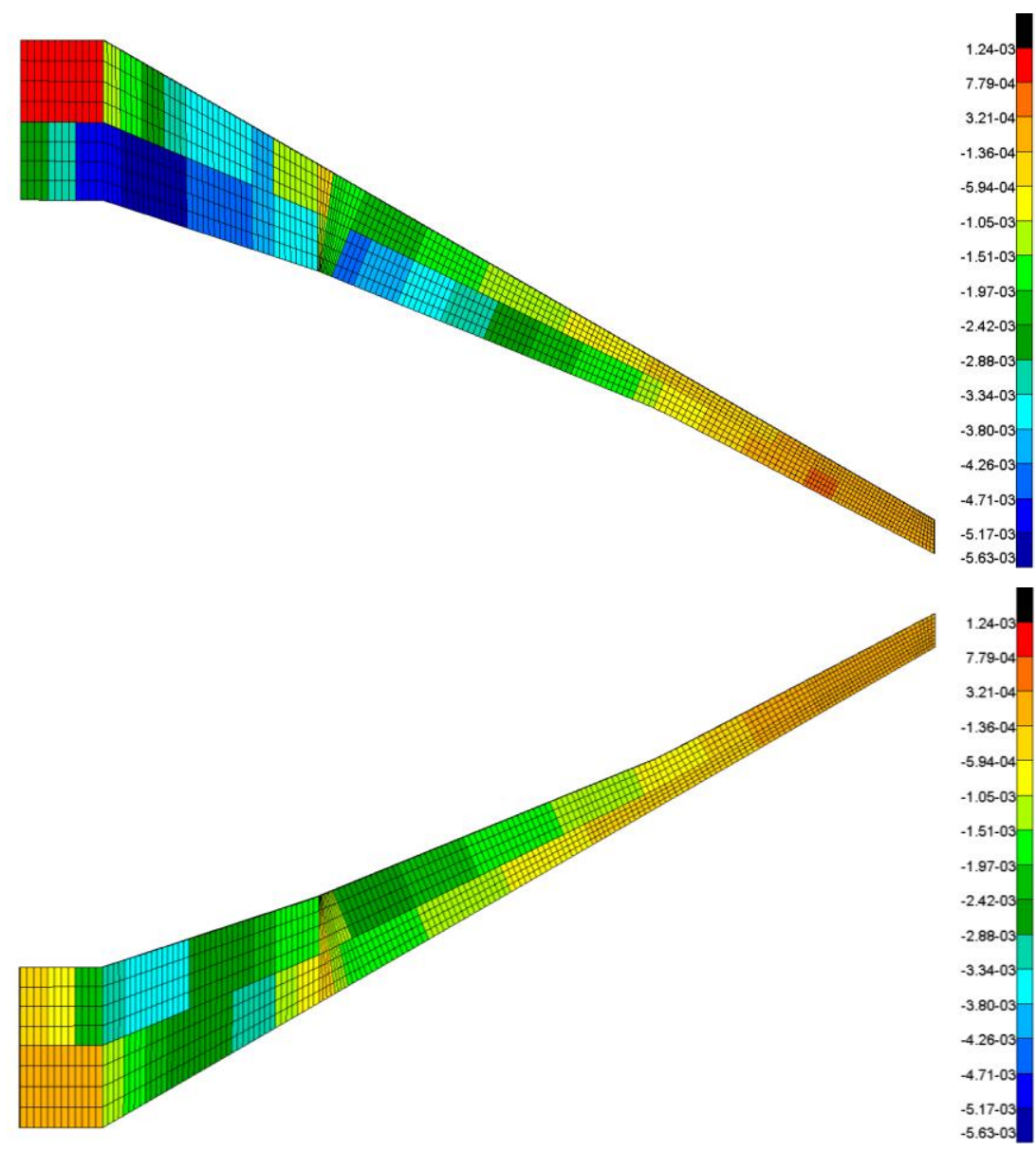

Fig. 22. Cross-sensitivity maps for the pressure drag coefficient (top: upper skin, bottom: lower skin).

\section{B. A Multi-Disicplinary Feasible implementation}

Next step will consider a more realistic parametrization of the wing shape including the additional control of the wing planform through variations of the chord sections and the span.

Our goal is to perform aero-structural optimizations considering a multi-point strategy for aerodynamic performance and a selection of 11 critical loads for certification. The same structural design model is considered (392 design variables) and about 19000 local design constraints (stress and buckling constraints) are defined for each load case. For such purpose we will pursue a fully integrated multi-disciplinary feasible strategy where a single optimizer manages all design variables and constraints at once (see Fig. 23). This approach allows taking into account multi-point aeroelastic optimizations and multiple sizing load cases simultaneously. In order to manage the huge number of structural design constraints, we will consider a gradient-based response surface reduced-ordermodel directly connected to the optimizer, thus avoiding the direct call to the high-fidelity aerodynamic and structural analysis codes. 


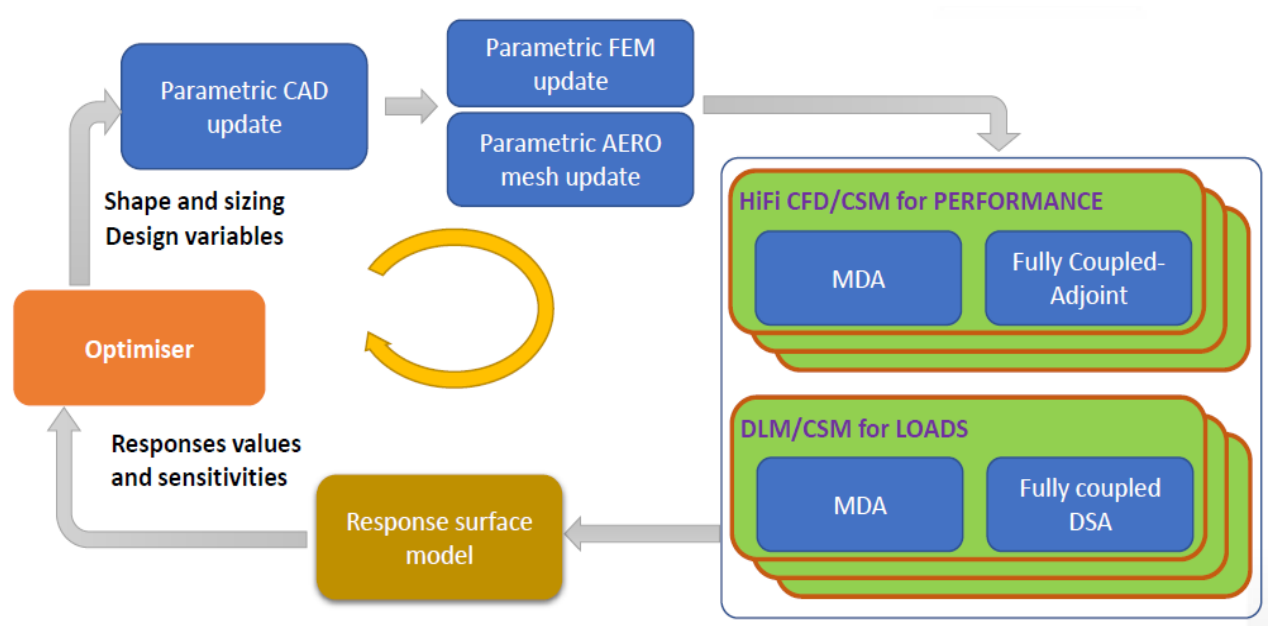

Fig. 23: Reference multidisciplinary feasible aero-structural optimization framework.

\section{Conclusions}

This work represents an important European collaboration between research institutes and industry in the aim of leveraging new capabilities for preliminary aircraft design. Despite the variability in the numerical approaches used by the different partners a very fair comparison for coupled gradient values has been achieved. More specifically the added benefit in terms of accuracy of considering flexibility in the computation of aerodynamic performance sensitivities has been demonstrated. In addition, it is now acknowledged by all partners that the fully linearized turbulence model should always be considered in gradient computations. An effective demonstration of these capabilities in terms of multi-point flexible optimization around an industrial-like configuration has been presented, paving the way to a more systematic study of the impact of the flexibility and the associated modelling on the final result and return time of the optimization process. Forthcoming investigations within the framework of the MADELEINE project will focus on this aspect by comparing purely aerodynamic, aeroelastic with rigid gradients and fully flexible approaches in the multi-point optimization of the considered test case, while virtually increasing the structural flexibility through the modification of the structural material properties. The aim is to clearly identify a trade-off between modelling complexity and performance benefits with respect to the degree of structural flexibility. Finally, an outlook on on-going developments for fully coupled aero-structure optimization has been also given: preliminary results of cross-sensitivities of the aerodynamic drag with respect to structural parameters have been presented and the envisaged strategy to efficiently manage the huge number of structural constraints has been clearly outlined.

\section{Acknowledgments}

We would like to thank Joël Brézillon and Romain Olivanti from the Flight Physics Capabilities Department of Airbus Operations SAS for providing us with the XRF-1 test case and the associated sensitivity results included in Fig. 7 and Fig. 8 for comparison. We would also thank them for fruitful discussions on the analysis of the results. The MADELEINE project has received funding from the European Union's Horizon 2020 research and innovation program under grant agreement No 769025 .

\section{References}

[1] Martins, J., Alonso, J., Reuther, J., and Reuther, J., “A Coupled-Adjoint Sensitivity Analysis Method for High-Fidelity Aero-Structural Design,” Optimization and Engineering, Vol. 6, 2005, pp. 33-62. doi:10.1023/B:OPTE.0000048536.47956.62.

[2] Kenway, G. K. W., and Martins, J. R. R. A., "Multipoint High-Fidelity Aerostructural Optimization of a Transport Aircraft Configuration," Journal of Aircraft, Vol. 51, No. 1, 2014, pp. 144-160. doi:10.2514/1.C032150. 
[3] Liem, R. P., Kenway, G. K. W., and Martins, J. R. R. A., "Multimission Aircraft Fuel-Burn Minimization via Multipoint Aerostructural Optimization," AIAA Journal, Vol. 53, No. 1, 2015, pp. 104-122. doi:10.2514/1.J052940.

[4] Brooks, T. R., Martins, J. R. R. A., and Kennedy, G. J., “Aerostructural Tradeoffs for Tow-Steered Composite Wings,” Journal of Aircraft, Vol. 57, No. 5, 2020, pp. 787-799. doi:10.2514/1.C035699.

[5] Merle, A., Ilic, C., Abu-Zurayk, M., Häßy, J., Becker, R.-G., Schulze, M., and Klimmek, T., "High-Fidelity Adjoint-based Aircraft Shape Optimization with Aeroelastic Trimming and Engine Coupling," EUROGEN 2019, Guimaraes, Portugal, 2019.

[6] Abu-Zurayk, M., Merle, A., Ilic, C., Keye, S., Goertz, S., Schulze, M., Klimmek, T., Kaiser, C., Quero, D., Häßy, J., Becker, R., Fröhler, B., and Hartmann, J., "Sensitivity-based Multifidelity Multidisciplinary Optimization of a Powered Aircraft Subject to a Comprehensive Set of Loads," AIAA AVIATION 2020 FORUM, American Institute of Aeronautics and Astronautics, 2020. doi:10.2514/6.2020-3168.

[7] Achard, T., Blondeau, C., and Ohayon, R., "High-Fidelity Aerostructural Gradient Computation Techniques with Application to a Realistic Wing Sizing”, AIAA Journal, Vol. 56, No. 11, 2018, pp. 4487-4499. doi:10.2514/1.J056736.

[8] Destarac, D., and van der Vooren, J., "Drag/thrust analysis of jet-propelled transonic transport aircraft; Definition of physical drag components," Aerospace Science and Technology, Vol. 8, No. 6, 2004, pp. 545-556. doi:10.1016/j.ast.2004.03.004.

[9] Méheut, M., Arntz, A. and Carrier, G. “Aerodynamic Shape Optimizations of a Blended Wing Body Configuration for Several Wing Planforms". 30th AIAA Applied Aerodynamics Conference 2012. 10.2514/6.2012-3122.

[10] Olivanti R. and Brézillon J. "On the Benefits of Engaging Coupled-Adjoint to Perform High-Fidelity Multipoint Aircraft Shape Optimization”. Submitted to AIAA Aviation Forum 2021.

[11] Cambier, L., Heib, S., and Plot, S., "The Onera elsA CFD Software: Input from Research and Feedback from Industry," Mechanics and Industry, Vol. 14, No. 3, 2013, pp. 159-174. doi:10.1051/meca/2013056. 